\title{
The significance of intraoperative fluorescence angiography in miniinvasive low rectal resections
}

\author{
Matej Skrovina ${ }^{1,2}$, Vladimir Bencurik², Lubomir Martinek ${ }^{2,3}$, Maria Machackova², Jiri Bartos², Petr Andel², \\ Erika Stepanova², Michaela Bunakova², Katerina Vomackova ${ }^{1}$ \\ ${ }^{1}$ Department of Surgery, Faculty of Medicine and Dentistry, Palacky University Olomouc, Olomouc, Czech Republic \\ ${ }^{2}$ Department of Surgery, Hospital Novy Jicin, Novy Jicin, Czech Republic \\ ${ }^{3}$ Department of Surgery, Faculty of Medicine, University of Ostrava, Ostrava, Czech Republic
}

Videosurgery Miniinv 2020; 15 (1): 43-48

DOI: https://doi.org/10.5114/wiitm.2019.84851

\begin{abstract}
Introduction: Anastomotic leak is a very serious complication in colorectal surgery. Tissue perfusion of the anastomosis plays an integral role in its multifactorial etiology. Fluorescence angiography using indocyanine green allows visualization of perfusion in real time.

Aim: To evaluate the effectiveness of intraoperative fluorescence angiography as a tool to decrease the incidence of anastomotic leak after laparoscopic or robotic low resection of the rectum for cancer.

Material and methods: Intraoperative fluorescence angiography was performed sequentially in 50 patients during low rectal resection for cancer with total mesorectal excision, primary anastomosis and protective ileostomy using laparoscopic or robotic technique. The results were compared to a historical control group of 50 patients with the same procedure without the use of fluorescence angiography.

Results: The patient sets were comparable in basic demographic and clinical parameters. Intraoperative visualization of perfusion by fluorescence angiography was achieved in all patients without unwanted side-effects. In 6 (12\%) patients, the resection line was adjusted based on the fluorescence angiography. The incidence of anastomotic leak was insignificantly lower in the group with fluorescence angiography (18\% vs. 10\%), which led to significantly shorter hospital stay. Other postoperative complications were comparable between the two groups.

Conclusions: Fluorescence angiography using indocyanine green is a safe and effective method with the potential of reducing anastomotic leak during minimally invasive low resection of the rectum for cancer.
\end{abstract}

Key words: anastomotic leak, fluorescence angiography, indocyanine green, rectal cancer.

\section{Introduction}

In colorectal surgery, constructing a perfect bowel anastomosis is one of the key moments of the surgical procedure. Healing complications - anastomotic leak - are among the most feared complications, which increase morbidity and mortality, worsen the prognosis of the patient and negatively affect his quality of life. Despite extensive research regarding the healing of the bowel anastomosis, attempts at identifying risk factors, implementation of modern surgical techniques and improving perioperative care, the incidence of this serious complication remains high. In the literature, anastomotic leak frequency ranges widely from $0.5 \%$ to $30 \%$ [1-5]. The frequency of its occurrence increases aborally with the highest values in the area of the distal rectum [4-6].

\footnotetext{
Address for correspondence

Prof. Lubomir Martinek MD, PhD, Hospital Novy Jicin, Novy Jicin, Czech Republic, phone: +420603500627 ,

e-mail: lubomir.martinek@post.cz
} 
Although the etiology of anastomotic leak in colorectal surgery is multifactorial, the vitality of the anastomosed segments of the bowel due to adequate perfusion represents one of the determining factors. However, the reliability of subjective evaluation of tissue vitality by the operating surgeon is limited [7-9]. Fluorescence angiography (FA) using indocyanine green (ICG) allows intraoperative visualization of the vascular network in real time and enables more objective evaluation of tissue perfusion.

\section{Aim}

The aim of our study was to evaluate the effectiveness of intraoperative fluorescence angiography using ICG in the near infrared spectrum (NIR) as a tool for decreasing the anastomotic leak rate (ALR) after laparoscopic or robotic rectal resection with total mesorectal excision for cancer.

\section{Material and methods}

The study comprised 50 sequentially included patients aged over 18 years of age, who underwent elective low rectal resection for cancer with total mesorectal excision, with primary double stapling end-to-end anastomosis and protective ileostomy by laparoscopic or robotic technique between August 2015 and February 2017. Intraoperative fluorescence angiography using indocyanine green in the near infrared spectrum was performed in these patients with the aim to objectively evaluate perfusion of the resection line. Patients with rectal cancer recurrence, those acutely operated or those with converted minimally invasive operations, as well as patients with a known allergy to iodine substances and pregnant or breastfeeding patients were excluded from the study. The results were compared with a control group of 50 electively operated patients, who underwent the identical non-converted minimally invasive procedure in the past without the use of fluorescence angiography. Patients from both groups were operated on by the same surgeons from the colorectal team. The study was a prospective, unicentric, non-randomized comparative study approved by the Institutional Ethics Committee.

Demographic and clinical patient data (gender, age, body mass index, ASA classification, comorbidities, nutrition status, medication with corticosteroids or smoking), data regarding the diagnosis of rectal cancer (TNM classification $-8^{\text {th }}$ edition, neoadjuvant radio/chemotherapy, distance of the cancer from the anal verge), characteristics of the surgical procedure (surgical technique, operation duration, blood loss, intraoperative complications), parameters of intraoperative fluorescence angiography (time necessary to achieve visualization, overall examination time, correction of the resection line based on visualized perfusion, side effects of ICG) and data regarding postoperative course (morbidity, mortality, anastomotic leak, postoperative complications according to the Clavien-Dindo classification, duration of hospital stay, resection of protective ileostomy) were studied prospectively.

To evaluate the perfusion of the resection line following mobilization and dissection of the bowel, indocyanine green (ICG-Pulsion $5 \mathrm{mg} / \mathrm{ml}$ Pulsion Medical Systems, Munich, Germany) was applied intravenously in a dose of $0.2 \mathrm{mg} / \mathrm{kg}$. Visualization of the vascular system was provided by the laparoscopic SPIES system (KARL STORZ GmbH \& Co. KG, Tuttlingen, Germany) or the Firefly robotic surgical system da Vinci Xi (Intuitive Surgical, Sunnyvale, CA, USA). Evaluation of perfusion of the determined resection line was performed individually by the surgeon, who then decided on the extent of correction of the resection line, if necessary. The colorectal anastomosis was examined in all patients using contrast examination (low irrigography) performed within 30 days of surgery. Anastomotic leak was classified according to the International Study Group of Rectal Cancer [10].

The primary aim of the comparative study was to evaluate the effectiveness of fluorescence angiography using ICG in decreasing the incidence of anastomotic leak of the colorectal anastomosis. Secondary aims were to evaluate the feasibility, safety and sensitivity of intraoperative fluorescence angiography using ICG in the NIR spectrum.

\section{Statistical analysis}

Descriptive statistical methods (mean, standard deviation, median, range and relative frequency) were used for data interpretation and statistical analysis. Student's t-test and the Mann-Whitney $U$ test were used for statistical comparison when the dependent variable was continuous, and the $\chi^{2}$ test in the case of categorical variables. A $p$-value less than 0.05 was considered to be statistically significant. 


\section{Results}

Basic demographic and clinical data of the compared sets of patients, including characteristics describing the cancer, are shown in Tables I and II.

There was a significantly greater representation of patients with hypertension and coagulopathy in the control group. There was no significant difference in nutrition status, location or stage of the cancer between the two groups. The rate of patients who underwent preoperative neoadjuvant therapy was also similar.

Characteristics of the surgical procedures are presented in Table III.

The two groups did not differ in the rate of laparoscopic and robotic procedures and were also comparable in most other studied surgical parameters, except for operation duration, which was significantly longer in the group with intraoperative FA. Fluorescence angiography in the near infrared spectrum extended the operating time by 6 min on average. Valid visualization was successfully achieved in all patients and we did not observe any side effects associated with intraoperative administration of indocyanine green.

In $6(12 \%)$ patients, intraoperative fluorescence angiography showed worsened perfusion and the surgeon decided to move the resection line $2-5 \mathrm{~cm}$ orally. Repeated examination of tissue vitality was subsequently performed in 5 patients and showed good perfusion of the oral bowel for anastomosis construction.

Data regarding the postoperative course, including complications, their severity and the frequency of anastomotic leak, are presented in Table IV.

The incidence and severity of postoperative complications did not differ between the two groups.

Table I. Demographic and clinical patient data

\begin{tabular}{|c|c|c|c|}
\hline Parameter & With fluorescence angiography $(n=50)$ & Without fluorescence angiography $(n=50)$ & $P$-value \\
\hline Age [years]: & & & 0.161 \\
\hline Mean \pm SD & $62.4 \pm 9.0$ & $65.0 \pm 9.4$ & \\
\hline Median (range) & $64.0(44-78)$ & $66.5(35-81)$ & \\
\hline Gender (male/female), $n$ (\%) & $34(68) / 16(32)$ & $29(58) / 21(42)$ & 0.300 \\
\hline BMI $\left[\mathrm{kg} / \mathrm{m}^{2}\right]$ : & & & 0.517 \\
\hline Mean \pm SD & $27 \pm 4$ & $27 \pm 5$ & \\
\hline Median (range) & $26(20-38)$ & $27(17-41)$ & \\
\hline ASA (I/II/III/IV), $n(\%)$ & $0(0) / 36(72) / 14(28) / 0(0)$ & $0(0) / 31(62) / 17(34) / 2(4)$ & 0.447 \\
\hline Diabetes, $n(\%)$ & $8(16)$ & $10(20)$ & 0.603 \\
\hline Corticosteroids, $n(\%)$ & $2(4)$ & $4(8)$ & 0.340 \\
\hline Hypertension, $n(\%)$ & $22(44)$ & $32(64)$ & 0.045 \\
\hline Atherosclerosis, $n(\%)$ & $8(16)$ & $4(8)$ & 0.218 \\
\hline Coagulopathy, $n(\%)$ & $0(0)$ & $4(8)$ & 0.041 \\
\hline $\begin{array}{l}\text { Smoker/ex-smoker/ } \\
\text { non-smoker, } n(\%)\end{array}$ & $14(28) / 5(10) / 31(62)$ & $5(10) / 7(14) / 38(76)$ & 0.070 \\
\hline
\end{tabular}

Table II. Characteristics of the tumor

\begin{tabular}{|c|c|c|c|}
\hline Parameter & With fluorescence angiography $(n=50)$ & Without fluorescence angiography $(n=50)$ & $P$-value \\
\hline TNM (0/I/II/III/IV), n (\%) & $9(18) / 21(42) / 9(18) / 8(16) / 3(6)$ & $10(20) / 13(26) / 11(22) / 15(30) / 1(2)$ & 0.261 \\
\hline $\begin{array}{l}\text { Preoperative radio/ } \\
\text { chemotherapy, } n(\%)\end{array}$ & $34(68)$ & $37(74)$ & 0.509 \\
\hline \multicolumn{3}{|c|}{ Distance from anal verge [mm]: } & 0.417 \\
\hline Mean \pm SD & $46 \pm 13$ & $46 \pm 15$ & \\
\hline Median (range) & $47(20-80)$ & $47(10-90)$ & \\
\hline
\end{tabular}


Table III. Operation data

\begin{tabular}{|c|c|c|c|}
\hline Parameter & With fluorescence angiography $(n=50)$ & Without fluorescence angiography $(n=50)$ & $P$-value \\
\hline $\begin{array}{l}\text { Operation technique } \\
\text { (laparoscop./robot), } n \text { (\%) }\end{array}$ & $35(70) / 15(30)$ & $37(74) / 13(26)$ & 0.656 \\
\hline Operation time [min]: & & & 0.018 \\
\hline Mean \pm SD & $242 \pm 48$ & $219 \pm 45$ & \\
\hline Median (range) & $240(158-410)$ & $210(130-365)$ & \\
\hline Duration of FA [min]: & & & - \\
\hline Mean \pm SD & $6 \pm 4$ & - & \\
\hline Median (range) & $5(3-20)$ & & \\
\hline Blood loss [ml]: & & & 0.441 \\
\hline Mean \pm SD & $52 \pm 47$ & $51 \pm 64$ & \\
\hline Median (range) & $30(5-200)$ & $30(300)$ & \\
\hline Blood transfusion, $n(\%)$ & $0(0)$ & $2(4)$ & 0.153 \\
\hline
\end{tabular}

In the group with fluorescence angiography, the frequency of the incidence of anastomotic leak decreased by $8 \%$ (18\% vs. $10 \%)$ and the duration of hospital stay was significantly shorter. Protective ileostomy was reversed in all patients in both groups.

\section{Discussion}

Despite significant advances of colorectal surgery in the past years, there has not been a radical decrease in the incidence of anastomotic leaks. In a meta-analysis and systematic review of 98 prospective studies in rectal surgery, the authors Paun et al. report that there is no significant difference in the frequency of leaks reported in publications before and after 2003 [11]. Therefore, much attention remains focused on the issue of bowel anastomosis healing.

The key requirements for uncomplicated healing are mechanical integrity and tissue vitality. Although intraoperative mechanical control of the integrity of the anastomosis has been standardly performed for a long time, objective evaluation of tissue vitality has only recently been introduced into clinical practice. In current colorectal surgery, a very promising method in this regard seems to be intraoperative

Table IV. Postoperative course

\begin{tabular}{|c|c|c|c|}
\hline Variable & With fluorescence angiography $(n=50)$ & Without fluorescence angiography $(n=50)$ & $P$-value \\
\hline $\begin{array}{l}\text { Postoperative } \\
\text { complications, } n(\%) \text { : }\end{array}$ & $15(30)$ & $18(36)$ & 0.580 \\
\hline Clavien-Dindo I-II & 7 & 11 & \\
\hline Clavien-Dindo III-IV & 8 & 7 & \\
\hline Anastomotic leak, $n$ (\%): & $5(10)$ & $9(18)$ & 0.163 \\
\hline Grade A & 2 & 8 & \\
\hline Grade B & 2 & 1 & \\
\hline Grade C & 1 & 0 & \\
\hline Mortality, $n(\%)$ & $0(0)$ & $0(0)$ & 1.000 \\
\hline Duration of hospital stay [days] & & & 0.013 \\
\hline Mean \pm SD & $8 \pm 4$ & $9 \pm 9$ & \\
\hline Median (range) & $7(5-24)$ & $6(4-50)$ & \\
\hline
\end{tabular}


fluorescence angiography using indocyanine green in the spectrum of near infrared light, which enables evaluation of tissue perfusion and vitality in real time. To date, published works focusing on this issue remain a heterogeneous assortment of open, laparoscopic and robotic procedures with different methodology. Most are prospective case series, while comparative case-control studies are rarely represented [9, 12-16]. Our work reports the results of comparing a relatively homogeneous set (elective low rectal resection for cancer with total mesorectal excision performed by minimally invasive technique without conversion) and with a fairly high number of patients as seen in larger studies. To date, the only similar study with a smaller number of patients was published in 2017 by Boni et al. [16].

Intraoperative visualization of tissue perfusion using ICG was successfully achieved in all patients in accordance with experiences reported in the literature [12, 17-21]. Technical execution slightly increased the duration of the surgical procedure. The administration of the indocyanine green, including repeating the dose, was completely safe, in agreement with other works [12, 17, 19, 21-25]. Based on our experience we consider intraoperative fluorescence angiography to be a feasible and safe method.

The effectiveness of the method is reflected in the frequency of modifications or changes to the original surgical procedure based on the performed fluorescence angiography. In our study, changes to the originally planned procedure after performing fluorescence angiography were made in $6(12 \%)$ patients. Similarly to other studies, the changes consisted in correcting the resection lines. The available literature reports corrections in resection lines ranging from $3.7 \%$ to $40 \%[12,17,20,22,25-27]$. Boni et al. report the relative frequency of changing the resection lines to be $4.7 \%$ in a very similar patient set [16]. In a recent systematic review, Blanco-Colino et al. report a correction of the planned resection line in $7.4 \%$ in a set of 555 patients with intraoperative fluorescence angiography using ICG during colorectal surgery [5].

The primary aim of the study was to evaluate the effectiveness of this method as a tool for decreasing the incidence of anastomotic leak after laparoscopic or robotic rectal resection with total mesorectal excision for cancer. In our patient set, we achieved an $8 \%$ decrease in the incidence of anastomotic leak $(18 \%$ vs. $10 \%$ ), although this result was not statistically significant, probably due to the small patient set (type II error). Similar comparative case control studies report a $4-12 \%$ decrease in the incidence of leak [12]. Boni et al. in the above-cited work did not observe any leak in the group of patients with intraoperative fluorescence angiography [16]. Analogous results, meaning without the presence of anastomotic leak, although in small and heterogeneous patient sets, were published by authors such as Sherwinter et al. [23], Ris et al. [19] or Martinek et al. [28]. The above-mentioned systematic review and meta-analysis by Blanco-Colino et al. indicate the positive potential of fluorescence angiography in reducing anastomotic leak in cancers of the colon and rectum [5]. Although the decrease in incidence of anastomotic leak after performing intraoperative FA was not statistically significant, it was reflected in a significantly shorter hospital stay.

Our results are limited mainly by the size of the patient set, the method (non-randomized study), and subjective evaluation of the perfusion image on the monitor. Despite all efforts, quantitative analysis of the fluorescence image is currently not generally available. Software analysis of the image, evaluation of the intensity of the fluorescence [29] or speed of its onset [30] requires standardized conditions which are difficult to achieve during regular operation. It may, however, be another step towards decreasing the incidence of this feared complication. Nonetheless, other questions remain. Should the perfusion test be performed only on the resection margins or also on the completed anastomosis? Can transanal examination of the low colorectal/coloanal anastomosis be useful? Another issue to consider is early follow-up using imaging methods to evaluate tissue vitality and monitoring levels of biomarkers [31], when there is suspicion of healing complications of the low anastomosis. A solution to at least some of these problems is studies with high quality methodology (not necessarily randomized studies) with a sufficient number of patients.

\section{Conclusions}

Fluorescence angiography using indocyanine green in the near infrared spectrum is a safe, easily performed, non-time-consuming and safe examination which allows intraoperative visualization of perfusion of the resection line and, based on our experience, contributes to a significant reduction of anastomotic leak after laparoscopic or robotic rectal resection with total mesorectal excision for cancer. 


\section{Conflict of interest}

\section{The authors declare no conflict of interest.}

\section{References}

1. Platell C, Barwood N, Dorfmann G, et al. The incidence of anas tomotic leaks in patients undergoing colorectal surgery. Colorectal Dis 2007; 9: 71-9.

2. Reinke CE, Showalter S, Mahmoud NN, et al. Comparison of anastomotic leak rate after colorectal surgery using different databases. Dis Colon Rectum 2013; 56: 638-44.

3. Damen N, Spilsbury K, Levitt M, et al. Anastomotic leaks in colorectal surgery. ANZ J Surg 2017; 84: 763-8.

4. McDermott FD, Heeney A, Kelly ME, et al. Systematic review of preoperative, intraoperative and postoperative risk factors for colorectal anastomotic leaks. Br J Surg 2015; 102: 462-79.

5. Blanco-Colino R, Espin-Basany E. Intraoperative use of ICG fluorescence imaging to reduce the risk of anastomotic leakage in colorectal surgery: a systematic review and meta-analysis. Tech Coloproctol 2018; 22: 15-23.

6. Caulfeld H, Hyman NH. Anastomotic leak after low anterior resection: a spectrum of clinical entities. JAMA Surg 2013; 148: 177-82.

7. Karliczek A, Harlaar NJ, Zeebregts CJ, et al. Surgeons lack predictive accuracy for anastomotic leakage in gastrointestinal surgery. Int I Colorectal Dis 2009; 24: 569-76.

8. Roesel C, Schachtrupp A, Schachtrupp A, Höer JJ. Intraoperative laser fluorescence angiography in colorectal surgery: a noninvasive analysis to reduce the rate of anastomotic leakage. Langenbeck's Arch Surg 2010; 395: 1025-30.

9. Jafari MD, Lee KH, Halabi WJ, et al. The use of indocyanine green fluorescence to assess anastomotic perfusion during robotic assisted laparoscopic rectal surgery. Surgical Endosc 2013; 27 3003-8.

10. Rahbari NN, Weitz J, Hohenberger W, et al. Definition and grading of anastomotic leakage following anterior resection of the rectum: a proposal by the International Study Group of Rectal Cancer. Surgery 2010; 147: 339-51.

11. Paun BC, Cassie S, MacLean AR, et al. Postoperative complications following surgery for rectal cancer. Ann Surg 2010; 251: 807-18.

12. Mizrahi I, Wexner SD. Clinical role of fluorescence imaging in colorectal surgery - a review. Expert Rev Med Devices 2017; 14 75-82.

13. Nachiappan S, Askari A, Currie A, et al. Intraoperative assess ment of colorectal anastomotic integrity: a systematic review. Surg Endosc 2014; 28: 2513-30.

14. Kudszus S, Roesel C, Schachtrupp A, et al. Intraoperative laser fluorescence angiography in colorectal surgery: a noninvasive analysis to reduce the rate of anastomotic leakage. Langenbeck's Arch Surg 2010; 395: 1025-30.

15. Kin C, Vo H, Welton L, et al. Equivocal effect of intraoperative fluorescence angiography on colorectal anastomotic leaks. Dis Colon Rectum 2015; 58: 582-7.

16. Boni L, Fingerhut A, Marzorati A, et al. Indocyanine green fluorescence angiography during laparoscopic low anterior resec- tion: results of a case-matched study. Surg Endosc 2017; 31: 1836-40.

17. Jafari MD, Wexner SD, Martz JE, et al. Perfusion assessment in laparoscopic left-sided/anterior resection (PILLAR II): a multi-institutional study. J Am Coll Surg 2015; 220: 82-92.

18. Ris F, Buchs NC, Morel P. Discriminatory influence of Pinpoint perfusion imaging on diversion ileostomy after laparoscopic low anterior resection. Colorectal Dis 2015; 17 Suppl 3: 29-31.

19. Ris F, Hompes R, Cunningham C, et al. Near-infrared (NIR) perfusion angiography in minimally invasive colorectal surgery. Surg Endosc 2014; 28: 2221-6.

20. Boni L, David G, Dionigi G, et al. Indocyanine green-enhanced fluorescence to assess bowel perfusion during laparoscopic colorectal resection. Surg Endosc 2016; 30: 2736-42.

21. Boni L, David G, Mangano A, et al. Clinical applications of indocyanine green (ICG) enhanced fluorescence in laparoscopic surgery. Surg Endosc 2015; 29: 2046-55.

22. Hellan M, Spinoglio G, Pigazzi A, et al. The influence of fluorescence imaging on the location of bowel transection during robotic left-sided colorectal surgery. Surg Endosc 2014; 28: 1695-702.

23. Sherwinter DA, Gallagher J, Donkar T. Intra-operative transanal near infrared imaging of colorectal anastomotic perfusion: a feasibility study. Colorectal Dis 2013; 15: 91-6.

24. Keller DS, Joshi HM, Rodriguez-Justo M, et al. Using fluorescence lymphangiography to define the ileocolic mesentery: proof of concept for the watershed area using real-time imaging. Tech Coloproctol 2017; 21: 757-60.

25. Skrovina M, Bencurik V, Holaskova E, et al. Fluorescence angiography in the detection of anastomotic perfusion during rectal and sigmoid resection - preliminary report. Rozhl Chir 2016; 95: 354-8.

26. Kawada K, Hasegawa S, Wada T, et al. Evaluation of intestinal perfusion by ICG fluorescence imaging in laparoscopic colorectal surgery with DST anastomosis. Surg Endosc 2017; 31: 106-9.

27. Grone J, Koch D, Kreis ME. Impact of intraoperative microperfusion assessment with Pinpoint Perfusion Imaging on surgical management of laparoscopic low rectal and anorectal anastomoses. Colorectal Dis 2015; 17 (Suppl. 3): 22-8.

28. Martinek L, Pazdirek F, Hoch J, et al. Intra-operative fluorescence angiography of colorectal anastomotic perfusion: a technical aspects. Rozhl Chir 2018; 97: 167-71.

29. Chang YK, Foo CC, Yip J, et al. The impact of indocyanine green fluorescence angiogram on colorectal resection. Surgeon 2018 Epub ahead of print. Doi:10.1016/j.surge.2018.08.006.

30. Son GM, Kwon MS, Kim Y, et al. Quantitative analysis of colon perfusion pattern using indocyanine green (ICG) angiography in laparoscopic colorectal surgery. Surg Endosc 2019; 33: 1640-49.

31. Dusek T, Orhalmi J, Sotona O, et al. Neopterin, kynurenine and tryptophan as new biomarkers for early detection of rectal anastomotic leakage. Videosurgery Miniinv 2018; 13: 44-52.

Received: 18.02.2019, accepted: 9.04.2019. 